Please quote as: Engel, C., Ebel, P. \& Leimeister, J. M. (2022). Cognitive automation. Electronic Markets. 


\title{
Cognitive automation
}

\author{
Christian Engel $^{1}\left[\right.$ [D $\cdot$ Philipp Ebel ${ }^{1} \cdot$ Jan Marco Leimeister ${ }^{1}$
}

Received: 12 March 2021 / Accepted: 6 December 2021

(C) The Author(s), under exclusive licence to Institute of Applied Informatics at University of Leipzig 2021

\begin{abstract}
Facilitated by AI technology, the phenomenon of cognitive automation extends the scope of deterministic business process automation (BPA) through the probabilistic automation of knowledge and service work. By transforming work systems through cognitive automation, organizations are provided with vast strategic opportunities to gain business value. However, research lacks a unified conceptual lens on cognitive automation, which hinders scientific progress. Thus, based on a Systematic Literature Review, we describe the fundamentals of cognitive automation and provide an integrated conceptualization. We provide an overview of the major BPA approaches such as workflow management, robotic process automation, and Machine Learning-facilitated BPA while emphasizing their complementary relationships. Furthermore, we show how the phenomenon of cognitive automation can be instantiated by Machine Learning-facilitated BPA systems that operate along the spectrum of lightweight and heavyweight IT implementations in larger IS ecosystems. Based on this, we describe the relevance and opportunities of cognitive automation in Information Systems research.
\end{abstract}

Keywords Cognitive automation $\cdot$ Knowledge work $\cdot$ Artificial intelligence $\cdot$ Machine learning $\cdot$ Cognition $\cdot$ Automation

JEL classification M15 $\cdot \mathrm{L} 21 \cdot \mathrm{C} 6$

\section{Introduction}

Artificial Intelligence (AI), which is facilitated by technological advancements in algorithms, computing power, and data storage during the last decades (von Krogh, 2018), offers novel ways of automating business processes, using Machine Learning (ML) as today's most prevalent instantiation of AI (Janiesch et al., 2021). Among other related terms, such as intelligent automation (Coombs et al., 2020) or hyper automation (Park, 2018), ML-facilitated business process automation (BPA) instantiates the novel phenomenon known by the term "cognitive automation": Cognitive automation aims at automating or augmenting tasks and processes seizing ML algorithms that facilitate processing structured and unstructured data, leading to probabilistic outcomes (Butner \& Ho, 2019; Lacity \& Willcocks, 2018b).

Responsible Editor: Rainer Alt

Christian Engel

christian.engel@unisg.ch

1 Institute of Information Management, University of St. Gallen, Müller-Friedberg-Strasse 8, 9000 St. Gallen, Switzerland
In BPA, this allows us to move beyond deterministic, rulebased automation as ML-facilitated BPA now takes over certain degrees of cognition, which poses the next evolutionary step beyond IT-facilitated BPA approaches such as workflow management (WfM) and robotic process automation (RPA) (Hofmann et al., 2020a, b; Lacity \& Willcocks, 2018b; Syed et al., 2020; Van Der Aalst et al., 2004). This opens up novel opportunities of automation in the field of knowledge and service work (Coombs et al., 2020). Facilitated by AI technology (AIT), respectively ML, cognitive automation now further extends the scope of automation through automating cognitive knowledge and work (Coombs et al., 2020; Drucker, 1993). Thus, we refer to the broader phenomenon of cognitive automation as seizing ML for automating knowledge and service work to realize value offered by AI, which is based on implementing artificial cognition that mimics and approximates human cognition in machines.

By transforming knowledge and service work through cognitive automation, organizations are provided with vast strategic opportunities to gain business value (Coombs et al., 2020). Businesses can further increase their levels of automation and/or reengineer their business processes 
such as deploying voice or chatbots for handling customer requests in service desk departments, performing automated criticality screenings of contracts in legal departments, or automatically translating texts or spoken language. Through these new automation opportunities, companies can gain competitive advantage by enhancing process efficiency and effectiveness (Zarkadakis, Jesuthasan, \& Malcolm, 2016). Therefore, cognitive automation is a strategic enabler of business transformation and productivity improvements, driving enterprise, customer, and employee value (Lacity \& Willcocks, 2018b, 2021). This is reflected in the market size of cognitive automation that in 2020 was estimated on a level between $\$ 50$ billion $\$ 150$ billion (Lacity \& Willcocks, 2021). Furthermore, BPA approaches such as ML-facilitated BPA, RPA, and WfM are predicted to evolve beyond company boundaries facilitating the automation of interorganizational transactions (Lacity \& Willcocks, 2021). This causes large impact on business ecosystems and electronic markets, ultimately impacting the future of work.

\section{Research need and approach}

In cognitive automation, various professions, disciplines and streams of research intersect, particularly the fields of Cognitive Science, Automation Research, and AI. All of these contribute different concepts helping to understand cognitive automation. Furthermore, systems that instantiate cognitive automation operate in and on larger complementary IS ecosystems. Thus, to make cognitive automation more predictable and explainable for both research and practice, we need to grasp the phenomenon as a whole, i.e., meticulously integrate different concepts and technologies that the cognitive automation ecosystem exhibits.

We deem the use of "cognitive automation" as most appropriate in this paper considering the multitude of existing terms that are used for describing novel ML-based approaches for automating knowledge and service work in front and back office processes: First, it appeals to the phenomenon that we intend to grasp, not merely to a technology, which is means-to-an-end. Second, we aim to provide a realistic view of the potential that our phenomenon of interest bears for front and back office automation. Thus, we limit the scope to cognition - i.e., "[...] all processes by which the sensory input is transformed, reduced, elaborated, stored, recovered, and used (Neisser, 2014, p.4) - rather than intelligence per se, which can be broadly defined as the ability that surrounds cognitive processes and facilitates them (Winfield, 2020). This shall allow for a fine-grained conceptualization of what is being automated by using ML as the term intelligence in its breadth poses an IS frontier rather than being currently realized.
To facilitate this knowledge creation process, we conducted a Systematic Literature Review (SLR) in 2020 (vom Brocke et al., 2015; Webster \& Watson, 2002). In that, our SLR's search scope is defined along the dimensions of search process, source, coverage, and techniques (vom Brocke et al., 2015): We applied a sequential search process using scientific databases as a source for our SLR. The database-driven approach helped us not neglect more recently published work, which is often the case in merely journal-based SLRs. Our literature search aims at reaching a representative coverage. We used a comprehensive set of search techniques to facilitate this representativity - keyword search, backward, and forward search.

Accordingly, we searched for literature in various databases relevant to the IS discipline (EBSCO, AISeL, Emerald, IEEE Xplore, JSTOR, and Science Direct). We used the search strings "cognitive automation" and combinations of "cognition", "automation", "artificial intelligence," and "machine learning". In addition to that, we conducted a backward and forward search on this basis to increase the representativity of our search scope. This helped us to integrate and structure distinct concepts as well as technology- and phenomenon-oriented perspectives on cognitive automation. Furthermore, this allowed us to derive a selection of themes that shall guide future research on cognitive automation in IS. We note here that we did not restrict the scope of our conceptualization merely to cognitive automation but also investigated its relationships to deterministic BPA approaches. We only deemed publications relevant to this fundamentals article that can be viewed as either "fundamental" components of the knowledge base on cognitive automation and its related or grounding concepts (i.e., wellcited) or that were highly suitable and timely for addressing our phenomenon of interest (i.e., mainly more recent publications). This selection process led to 37 papers that we thoroughly analyzed.

In the remainder of this paper, we first elaborate on the constituting concepts of cognitive automation to shed light on its grounding. In addition, we provide an overview of the major BPA approaches such as workflow management, robotic process automation, and Machine Learning-facilitated BPA while emphasizing their complementary relationships in the ecosystem of BPA solutions. On this basis, we provide an integrated conceptualization of the phenomenon of cognitive automation. Finally, we describe the relevance and opportunities of cognitive automation in research on Information Systems (IS) against the backdrop of electronic markets (see also Alt \& Klein, 2011).

Overall, this paper shall serve as a terminological basis and a unified conceptual lens for advancing our understanding of cognitive automation by relating it to and delimitating it from rule-based automation research (e.g., Hofmann et al., 2020a, b). Furthermore, we intend to clarify the positioning 
of cognitive automation at the intersection between BPA and AI by specifically considering its most prevalent technical implementations, i.e. ML and Deep Learning (e.g., Janiesch et al., 2021). Ultimately, this shall contribute to a more realistic, less hype- and fear-induced future of work debate on cognitive automation.

\section{Conceptual foundations of cognitive automation}

\section{Cognition and automation}

Cognition has been studied in a variety of research fields. Thus, there is no general or universal definition of cognition that fits the purpose of each discipline at once (Gershenson, 2003). For this paper, we refer to a definition of cognition that deciphers the process of developing knowledge and understanding. This deems appropriate as we link the concept of cognition to the context of task and process automation: "'Cognition' refers to all processes by which the sensory input is transformed, reduced, elaborated, stored, recovered, and used. [...] Such terms as sensation, perception, imagery, retention, recall, problem-solving, and thinking, among many others, refer to hypothetical stages or aspects of cognition" (Neisser, 2014, p.4). Furthermore, we narrow down our terminological scope of cognition to the notions of human and artificial cognition (Gershenson, 2003). Human cognition is the most intensely covered stream of cognitive science. Artificial cognition refers to a phenomenon instantiated by systems often called cognitive machines such as expert systems, robots, and software agents built by us humans to mimic human cognition (Gershenson, 2003; Nobre et al., 2009). In the vein of artificial cognition, cognitive machines are used to approximate and mimic what Kahneman (2015) calls system 2 thinking, which is logical, rather effortful, and conscious, and follows the probability theory paradigms. Humans are superior in system 1 thinking, which refers to affective, fast, emotional, ad-hoc, stereotypic, subconscious thinking that is primarily related to human intuition and creativity (Kahneman, 2015). IS researchers have explored the design, use and integration of cognitive machines in distinct socio-technical contexts. This shall extend and advance both individual and organizational capabilities in terms of information processing, decision-making, and problem-solving capacities through human-computer interaction (Nobre et al., 2009). In the realm of artificial cognition, seizing a cognitive functions lens, recent research finds cognitive machines to outperform humans in perceiving and reasoning, while humans still play a dominant role in the cognitive functions of decision-making, learning, and planning (Stohr \& O'Rourke, 2021). In this vein, Hofmann et al. (2020a) derive a set of AI functions from cognitive abilities - in particular, perceiving, feature extraction and identification, reasoning, predicting, decisionmaking, generating, and acting. Similarly, Rai et al. (2019) also use the cognitive function lens to explain AI as the ability to perform cognitive functions, which relates to the differentiation between intelligence and cognition that we introduced earlier in this paper. This explains why we use the concepts of cognitive functions as well as artificial cognition as the conceptual glue for grasping cognitive automation in this paper.

Automation refers to the full or partial "execution by a machine agent (usually a computer) of a function that was previously carried out by a human" (Parasuraman \& Riley, 1997, p.231), and is viewed to be a possibility for humans to extend their tool and machine capability. In automation research, three groups of automation are generally distinguished: mechanical automation, information and control automation, and combinations of these two (Fasth-Berglund $\&$ Stahre, 2013). While mechanical automation focuses on the automated execution of physical tasks and processes, information and control automation refers to non-physical automation, such as in business tasks and processes.

Cognitive automation focuses on automating knowledge and service work (Coombs et al., 2020; Drucker, 1993) and thus necessarily involves the automation of information and control, which is a particularly important perspective on automation in IS. From an information and control perspective, there are four types of automation structured along the stages of human information processing: (1) automated information acquisition, (2) automated information analysis, (3) automated decision selection, and (4) automated action implementation (Parasuraman et al., 2000). Along the different types of automation, the degree of automation can continuously vary. Thus, cognitive automation is a matter of degree, not of kind.

It is important to emphasize that even high levels of automation should not be confused with the term of "autonomy", although the concepts are related. Autonomy refers to an entity's or agent's ability to act self- determined and independently (Janiesch et al., 2019). In that, automation poses a necessary condition for machine autonomy, which can be reached if all cognitive functions described above are performed by a machine without human intervention and responsibility (Janiesch et al., 2019).

In this paper, we focus on ML-facilitated BPA, which we refer to as the most prevalent instantation of the phenomenon of cognitive automation. BPA uses process and task descriptions for guiding the performance of business activities (Hofstede et al., 2010). BPA is a part of the field of Business Process Management, which has its roots in the field of WfM (W. M. P. van der Aalst et al., 2018). Especially, in recent years RPA has evolved as a novel form of BPA, which uses an 'outside-in', approach where the existing 
information systems remain unchanged aiming for quick wins with little investments (W. M. P. van der Aalst et al., 2018). RPA is based on processing rules leading to deterministic outcomes. Thus, we subsumed RPA as one form of deterministic automation approaches in the same vein as WfM, which instantiate the phenomenon that we call "rulebased automation" in this paper.

\section{Delimitating artificial intelligence, machine learning, and cognitive automation}

AI refers to all techniques facilitating machines to mimic human behavior, i.e., reproducing or excelling over human decision-making with the goal to solve complex tasks with minimal human intervention or completely independently (Russell \& Norvig, 2021). For the last years, AI has been one of the fastest growing research streams within the IS domain and has become one of the "hot topics" in business and industry practice (Abdel-Karim et al., 2021; Jordan \& Mitchell, 2015). This is facilitated by steep developments in algorithms, computing power, and data storage (von Krogh, 2018). AI has been categorized in narrow AI and Artificial General Intelligence (Gubrud, 1997). This categorization has been widely applied in research to clearly define the scope of analysis (e.g., Engel et al., 2021a). Thus, when referring to $\mathrm{AI}$ in this paper, we mean narrow $\mathrm{AI}$ rather than Artificial General Intelligence. Against the backdrop of contributing to the fundamentals of cognitive automation, our reasoning is the following: While the former has already proven its feasibility in the real world, the latter is still more in the focus of public debates than being technically realized. In that, narrow AI refers to an AI exhibiting equal or better capabilities than a human in a specific domain of tasks, whereas an Artificial General Intelligence is posed to exhibit equal or better capabilities than a human in any domain of tasks (Gubrud, 1997). To further increase the conceptual clarity of cognitive automation, we provide an overview of the definitions of AI, ML, as well as cognitive automation in Table 1. Furthermore, we integrate the relationships, delimitations, and terminological scope of these concepts to further grasp our phenomenon of interest.

Nowadays, the most prevalent technology used for designing, creating, and running cognitive automation revolves around ML as a concrete instantiation of AI-specific technological advancements (Janiesch et al., 2021). However, ML is not the only implementation of AI and in the future, there might be novel more advanced technologies on the rise such as "Quantum Deep Learning", which refers to algorithms currently being developed based on deep quantum networks that are facilitated by deep quantum information processors consisting of photonic circuits (Pramod et al., 2021). This is closely related to the so-called "AI effect" (Haenlein \& Kaplan, 2019), which describes the tendency of humans to only call something AI that is yet not feasible. Once it is technically feasible, they do not call it AI anymore but simply computing. To grasp the most relevant implementation of cognitive automation here, we briefly introduce the basic concepts of ML.

The following definition of ML grasps its underlying mechanism: ML refers to building computer programs that improve automatically based on improving a measure of performance when executing a task through training experience (Jordan \& Mitchell, 2015). Less mechanistically speaking, ML is a set of techniques that revolve around training computers to perform tasks based on prior examples, not only based on predefined rules that are explicitly programmed (Wilamowski \& Irwin, 2018). The notion of "learning" in ML can thus be described as inferring models to explain observed data, which shall enable a machine to make predictions about future data, and to make decisions based on these predictions (Ghahramani, 2015). Generally, there are two main types of ML approaches: Supervised (labeled data) vs. unsupervised (unlabeled data) ML (Russell \& Norvig, 2021). ML systems are trained rather than programmed, which refers to the goal of learning patterns

Table 1 Integrated phenomenon-oriented definition and delimitation of cognitive automation

\begin{tabular}{|c|c|c|}
\hline Artificial Intelligence & Machine Learning & Cognitive Automation \\
\hline $\begin{array}{l}\text { Artificial Intelligence refers to all techniques } \\
\text { facilitating machines to mimic human behav- } \\
\text { ior, i.e., reproducing or excelling over human } \\
\text { decision-making with the goal to solve com- } \\
\text { plex tasks with minimal human intervention } \\
\text { or completely independently } \\
\text { (Russell \& Norvig, 2021) }\end{array}$ & $\begin{array}{l}\text { Machine learning refers to building computer } \\
\text { programs that improve automatically based } \\
\text { on improving a measure of performance } \\
\text { when executing a task through training expe- } \\
\text { rience (Jordan \& Mitchell, 2015) }\end{array}$ & $\begin{array}{l}\text { Cognitive Automation refers to seizing ML for } \\
\text { automating cognitive knowledge and service } \\
\text { work to realize value offered by AI, which is } \\
\text { based on implementing artificial cognition } \\
\text { that mimics and approximates human cogni- } \\
\text { tion in machines }\end{array}$ \\
\hline \multicolumn{3}{|l|}{ Conceptual Relationships and Scope } \\
\hline $\begin{array}{l}\text { Superordinate phenomenon: Includes } \\
\text { machines performing existing and new tasks } \\
\text { and/or processes, producing decisions and/ } \\
\text { or solutions }\end{array}$ & $\begin{array}{l}\text { Technological means: Machine Learning is } \\
\text { the most prevalent technical implementation } \\
\text { of (narrow and weak) AI }\end{array}$ & $\begin{array}{l}\text { Intersection of AI and BPA: Defined by the } \\
\text { purpose of creating value through automating } \\
\text { or augmenting existing cognitive tasks and/or } \\
\text { processes using ML }\end{array}$ \\
\hline
\end{tabular}


and relationships from previous examples (Bishop, 2006). By automating the task of building analytical models, cognitive functions can be performed by machines such as object detection, speech and image recognition, or natural language processing (Janiesch et al., 2021). In recent years, an intense driver of advancements in ML has been its subfield of Deep Learning (Pramod et al., 2021). Deep Learning is an ML concept based on artificial neural networks, which have been developed according to information processing in biological systems replicating or at least approximating human cognition (LeCun et al., 2015). These advancements in ML have facilitated the pervasive impact on business and personal lives by providing increasingly human-like cognitive capacities, which also significantly shapes networked interactions in electronic markets (Janiesch et al., 2021).

Cognitive Automation refers to seizing ML for automating knowledge and service work to realize value offered by $\mathrm{AI}$, which is based on implementing artificial cognition that mimics and approximates human cognition in machines. This leads to a new allocation of cognitive functions - i.e., perceiving, reasoning, decision-making, learning, and planning - between humans and machines (Stohr \& O'Rourke, 2021). While the phenomenon of cognitive automation is driven by the purpose of automating existing cognitive tasks and processes, $\mathrm{AI}$ is less specific in its purpose as it comprises both existing and potentially new tasks and/or processes.

\section{Overview of business process automation approaches}

To clearly explain the relation between the broader concept of cognitive automation and the narrower field of BPA, we provide an overview of BPA approaches such as WfM, RPA, and ML-Facilitated BPA in Table 2. This shall serve as a foundation for clearly delimitating cognitive automation from rule-based automation approaches in the field of software robots in the next chapter (Hofmann et al., 2020a, b; Kroll et al., 2016).

WfM as the early beginnings and still widespread and valid foundation of BPA can be viewed as an answer to the call for seizing the opportunities of IT for managing business processes in an automated manner (W. Van Der Aalst et al., 2004). A prominent example includes processing of customer payments through multiple departments of a bank in an end-to-end manner - known by the name of straight-through processing. In WfM, business processes are designed on a higher level of abstraction before the design and implementation of the respective IS and organizational structures and processes is pursued (W. Van Der Aalst et al., 2004). WfM is instantiated as generic software packages that take on the information logistics of business processes

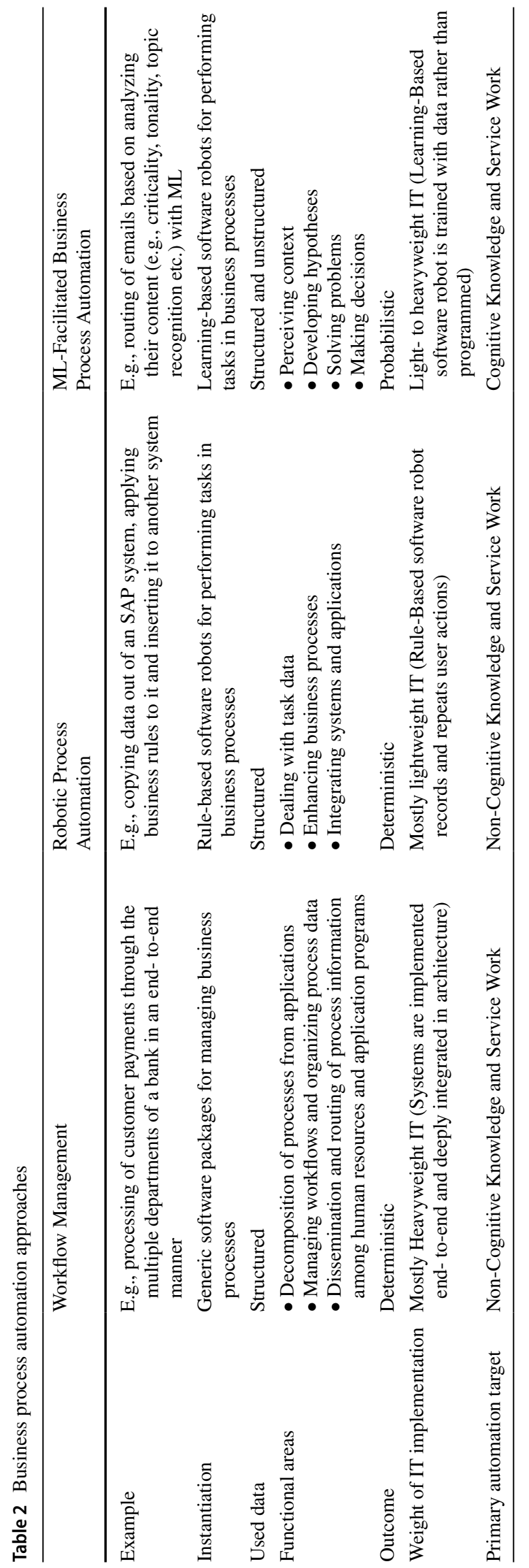


using structured data about these processes. Functional areas include the decomposition of processes from applications, managing workflows, organizing the process data, and disseminating and routing process information among human resources and application programs (W. Van Der Aalst et al., 2004). In that, the outcomes of these forms of BPA are deterministic as all business process rules are predefined by a human entity and follow if-then structures. In that, WfM primarily targets the automation of non-cognitive knowledge and service work. As WfM is implemented in an end-toend manner with a high level of IT architecture integration, it is considered as heavy-weight IT rather than lightweight IT. While lightweight IT refers to "[a] knowledge regime, driven by competent users' need for solutions, enabled by the consumerisation of digital technology and realized through innovation processes", heavyweight IT refers to "[a] knowledge regime, driven by IT professionals, enabled by systematic specification and proven digital technology and realized through software engineering" (Bygstad, 2017, p. 182).

In recent years RPA has evolved as a novel form of BPA, which uses a lightweight IT, “outside-in" approach where the existing information systems remain unchanged aiming for quick wins with little investments (W. M. P. van der Aalst et al., 2018). RPA refers to "using software to automate tasks previously performed by humans that use rules to process structured data to produce deterministic outcomes" (Lacity \& Willcocks, 2018a, p.24).

Rule-based software robots in RPA represent software agents that can mimic user actions in order to interact with software systems, thus reducing the workload of the human agents (Syed et al., 2020). For instance, RPA can be used to record and repeat user actions such as copying data out of an SAP system, applying business rules to it and inserting it to another system. Hofmann et al. (2020a, b) identify three main functional areas that summarize the core capabilities of RPA: (1) dealing with data, (2) process enhancement, and (3) integration of systems. In that, RPA is usually applicable when levels of standardization, maturity, transaction volume, and existence of business rules are all high (Lacity \& Willcocks, 2018b). According to other criteria, rule-based routine tasks with few exceptions and little or no cognitive reasoning are most suitable for RPA (Asatiani \& Penttinen, 2016; Zarkadakis et al., 2016). Other archetypical RPA tasks include the transfer of data from one software system to another such as taking inputs from client emails or excel spreadsheets, processing the information in a deterministic manner, and then entering the results into some other software systems, such as Customer Relationship Management systems (Zarkadakis et al., 2016). Thus, rule-based software robots mainly access information systems via the frontend (W. M. P. van der Aalst et al., 2018). However, RPA can vary in its level of IT infrastructure integration ranging from software robots merely screen-scraping user interfaces to being deeper embedded in the underlying business logic (W. M. P. van der Aalst et al., 2018). Still, RPA is generally viewed as lightweight IT that can be implemented in a userdriven, self-service, low-code manner (Bygstad, 2017).

While RPA relies on so-called rule-based software robots that operate according to predefined rules, ML- facilitated BPA approaches rely on so-called learning-based software robots that develop experience from data (Kroll et al., 2016). Learning-based software robots rely on the ability to "[...] build patterns, and relationships among data, and apply knowledge to solve problems or pose additional pertinent questions." (Lacity \& Willcocks, 2018a, p.26). For instance, ML-facilitated BPA can be used for routing emails based on analyzing their content (e.g., criticality, tonality, topic recognition etc.). Thus, machine perception has been found to be one prerequisite for ML-facilitated BPA approaches and thus for the broader phenomenon of cognitive automation (Bruckner et al., 2012). Furthermore, software is needed to mimic human activities such as perception, developing hypotheses, and reason upon them to perform judgementintensive tasks (Rainey et al., 2017), which require action supported by context in a manner of human rationale (Poosapati et al., 2018). For instance, ML-facilitated BPA can be used to automate (parts of) call centers through automatically understanding natural language of callers, developing a machine perception of meaning and mimicking the reasoning about possible action decisions, such as automated routing of an incidents to the right resolver team (Poosapati et al., 2018).

This means while RPA is programmed deterministically, ML-facilitated BPA tools need to learn from examples of prior data in a domain of interest (Rainey et al., 2017). Consequently, these systems need to take into account vast amounts of details (including structured and unstructured data) as more business processes require analysis and insights that allow controlling them beyond rule-based execution or prefitted controllers (Bruckner et al., 2012). Thus, as explained earlier, in the realm of BPA, the phenomenon of cognitive automation is particularly instantiated by the application of technologies from the realm of AI, i.e., ML, which includes Deep Learning. These technologies are used to create machines that perform tasks and processes based on context by applying, for instance, natural language processing or image recognition, etc. (Poosapati et al., 2018).

Against this backdrop, ML-facilitated BPA solutions are more versatile in terms of their categorization as lightweight or heavyweight IT (Bygstad, 2017), which means that software needed to instantiate the phenomenon of cognitive automation is more variable in its particular ease of use and its modularity than RPA.

Accordingly, depending on the particular use-case, there are pretrained plug-and-play solutions that can be easily 
added to existing applications, whereas implementing MLfacilitated BPA solutions can also lead to large scale projects of gathering data, readjusting business logics, reorganizing databases, and (re)establishing respective data pipelines for processing highly voluminous, fast, and heterogeneous data.

\section{An integrated conceptualization of cognitive automation}

Building up on and extending the conceptual and terminological foundations presented in the previous section, we present an integrated conceptualization of cognitive automation in this chapter (see also Fig. 1). We explicitly demonstrate how technology, phenomena and automation targets are related to reach an integrative multi-facetted view of cognitive automation. Furthermore, we point out the complementary relationship between rule-based and cognitive automation approaches for non-cognitive and cognitive knowledge and service work.

As already emphasized, automating tasks and processes has a longstanding tradition in IS research. However, classic, rule-based automation research can be described as rather deterministic: From a rule-based automation perspective, decisions are still made, and resolutions are still chosen by human beings, seizing and applying their human cognition (Ogiela \& Ogiela, 2014). This is due to the fact that rules are implemented ex-ante determining the automation outcome. Accordingly, this means that even if a deterministic system operates seemingly autonomously, it does still not make decisions or provides solutions for problems as its outcomes are predefined and thus deterministic. Much more, the human still conducts decision making and problem solving, even though this occurs before the task or process is carried out in an automated manner. We subsume these deterministic BPA approaches, i.e., RPA and WfM, under the term "rule-based automation" as a sub phenomenon of
Information and Control Automation (see also Phenomena in Fig. 1). The primary automation target of rule- based BPA is non-cognitive knowledge and service work, which for instance includes swivel-chair tasks such as copying and pasting data from one system to another one (see also Targets in Fig. 1).

In cognitive automation, certain degrees of cognition are taken over and approximated by machines that provide two types of outputs - decisions and/or solutions (von Krogh, 2018). While decisions refer to conclusions that are reached through the deliberation of algorithms based on the data available, solutions are defined as alternative courses of action for problem resolution (von Krogh, 2018). Thus, cognitive automation is a phenomenon that incorporates the approximation and mimicking of a certain degree of human cognition by machines (i.e., artificial cognition, especially system 2 thinking (Kahneman, 2015)) with the primary goal to target the automation of cognitive knowledge and service work (see also Target in Fig. 1) (Coombs et al., 2020; Drucker, 1993). To facilitate the automation of cognitive knowledge and service work, cognitive automation operates probabilistically rather than deterministically (see also Phenomena in Fig. 1).

From a technology-oriented lens, the particular Information Technology (IT) that facilitates the phenomenon of cognitive automation exhibits specific characteristics required to transport the capabilities for "producing" decisions or solutions. To seize the automation potential created by the rise of AI, nowadays cognitive automation predominantly relies on ML (Butner \& Ho, 2019; Lacity \& Willcocks, 2018b), which we introduced in the conceptual foundations section of this paper. To incorporate the respective advancements in algorithmic capabilities, computing power, and data storage, we use AI Technology (AIT) as the technology-oriented label to describe the particular subset of Information Technology (IT) that specifically serves as the technological facilitator of the phenomenon of cognitive automation (see also
Fig. 1 Integrated conceptualization of cognitive automation

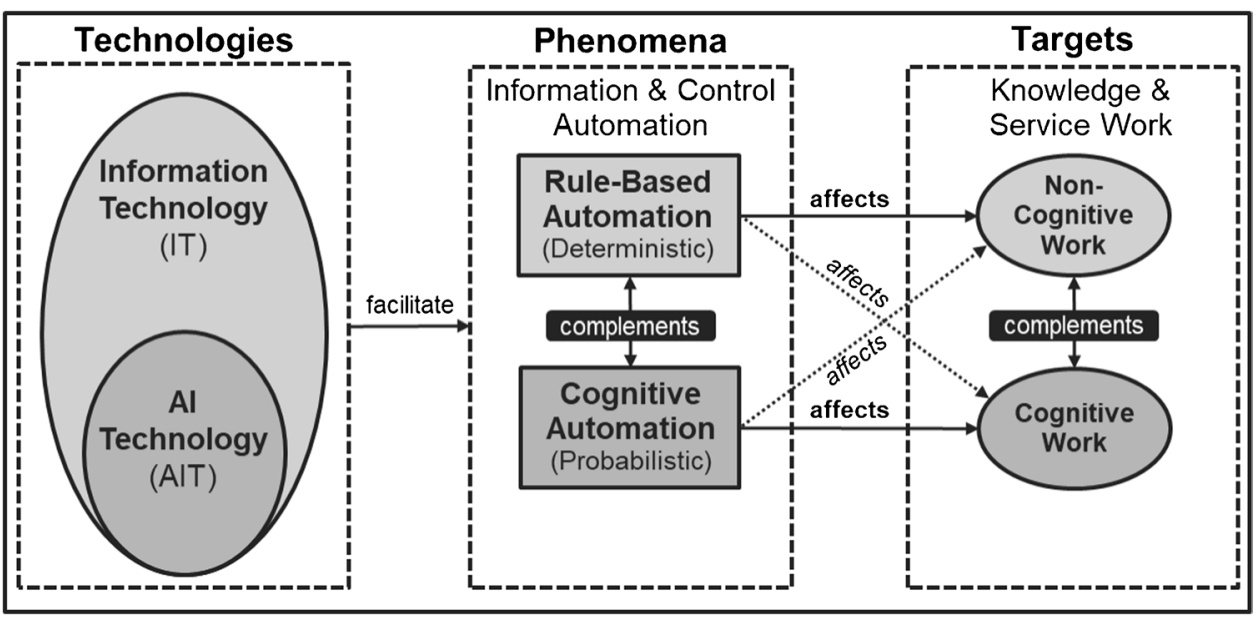


Technologies in Fig. 1). The delimitation of phenomena and technologies should not be interpreted dichotomously but continuously, which provides researchers with the opportunity to contribute to theoretical work explaining and guiding the journey on this continuum.

Even though rule-based automation operates deterministically and cognitive automation probabilistically, they are nevertheless highly synergistic and complementary phenomena (see also complement in Fig. 1) that form a symbiosis if the underlying technology is orchestrated correctly (Lacity \& Willcocks, 2018b, 2021). These complementary relationships impact the way how non-cognitive and cognitive knowledge and service work can be automated in an integrated manner. We have visualized this reasoning in Fig. 1 through the "complements" boxes to show that both the phenomena as well as the automation targets are interdependent as they imply a need to be orchestrated and interwoven efficiently and effectively for achieving the intended business value - i.e., rule-based automation indirectly affects cognitive and cognitive automation also indirectly affects non-cognitive knowledge and service work. For instance, when automating the handling of customer request that reach a service center through channels such as email or phone, cognitive automation, e.g., image, text or voice recognition algorithms, can be used to preselect an appropriate resolution option and provide a routing decision to a particular rule-based automation software agent that executes the remaining steps in a deterministic manner (Lacity \& Willcocks, 2018b). Such complementary relationships between automating cognitive work and automating non-cognitive work also exist between automated parts of work and non-automated parts that are still conducted by humans. Thus, research has identified multiple strategies of automating knowledge and service work as proposed by Sampson (2021): the augmentation, deskilling, automation, and centralizing strategy. The selection of the particular strategy depends on the degree of required creative skills and required interpersonal skills of employees when performing a task or process. These also apply to cognitive automation and rule-based automation of knowledge and service work: The augmentation strategy applies to those tasks and processes that require both high interpersonal and high creative skills (Sampson, 2021). Here, cognitive automation would support a professional in task performance. The deskilling strategy applies when the required level of creativity is not high but still a high amount of interpersonal skills is needed to perform the task (Sampson, 2021). Thus, this interactive work should be shifted to less expert workers at lower costs (Davenport \& Kirby, 2016). These lower skilled employees should be simultaneously equipped with cognitive automation tools to keep up the level of service quality. The automation strategy should be applied when both the required creative and interpersonal skill levels are low in a task or process (Sampson, 2021). Thus, these tasks or processes can ideally be fully carried out by cognitive automation in combination with rule-based automation. The centralizing strategy refers to a situation where a task requires high levels of creative expertise but low levels of interpersonal skills. Here, a trained professional should be decoupled from customers, and customer interactions should be automated (Sampson, 2021).

\section{Relevance and research opportunities}

Cognitive automation impacts both organizations and IS ecosystems, which requires companies to approach cognitive automation initiatives in a strategic manner (Hofmann et al., 2020a, b). Here, in line with other researchers, we emphasize that ML does not pose a "silver bullet" to BPA but that the novel opportunities come hand in hand with new challenges (Herm et al., 2021, p.302). This is reflected in the matter of fact that besides the great potential cognitive automation offers, only 26 percent of potential adopter organizations state to have particular systems in place, which is rooted in the still comparably high price of cognitive automation tools, the required amounts of data, and the insecurity of organizations due to the unpredictability and probabilistic character of outcomes (Lacity \& Willcocks, 2018a). Combined with the challenge of balancing lightweight and heavyweight implementation of cognitive automation (in symbiosis with other forms of BPA) in IS ecosystems, this offers vast opportunities for researchers. Here, we propose a selection of themes that are likely to drive research on cognitive automation in IS.

\section{Ecosystem- and platform-oriented research opportunities}

Electronic markets are becoming more and more data-driven, which is facilitated by developments in AI, ML, as well as the pervasiveness of data being produced and being accessible (Selz, 2020). In electronic markets, we are faced with a variety of distinct stakeholder touchpoints, such as websites, apps, and social media platforms, which create large volumes of structured, unstructured, and heterogeneous data (Janiesch et al., 2021). To create, deliver and capture value from this data through cognitive automation, organizations will need to overcome challenges that occur on a broader IS ecosystem level, which consists of different applications (Hofmann et al., 2020a, b). This requires orchestrating software robots (i.e., cognitive automation and RPA systems) and other information systems such as WfM systems as well as applications that vary in their "weight" of IT implementation. In this paper, we described how organizations can benefit from the symbiosis of 
ML-facilitated BPA, RPA and WfM against the backdrop of the spectrum of lightweight and heavyweight IT. To leverage this potential, organizations face the need to balance highly integrated and structured solutions vs. so-called meshworks created by heterogeneous technological users and agents (Bygstad, 2017). Here, research and practice efforts are required to manage task sequences across applications in a manner of automated learning (Herm et al., 2021).

Furthermore, we pointed out earlier that to maximize value from BPA requires organizations to manage the orchestration of the complementary kinds of BPA approaches - i.e., WfM, RPA, and ML-facilitated BPA. As a consequence, this has led to a rise in third-party developers, which creates the challenge for organizations to balance the interconnection of internal and external, heavyweight and lightweight IT. Currently, it can be observed that this increasingly leads to the establishment of so-called platform systems, which pose the facilitating centers of larger ecosystems (Bygstad, 2017). This is reflected in cognitive automation systems operating in and on larger complementary IS ecosystems such as using training data sets and open-source ML models provided by Google, or platforms such as Microsoft Azure, BluePrism, Automation Anywhere, or UiPath, as well as by accessing computing power through the cloud (e.g., Amazon Web Services), which refers to cloud-based platformization (Benlian et al., 2018). Especially, the emergence of novel AI as a service (AIaaS) ecosystems is largely impacting the phenomenon of cognitive automation. AIaaS is a combination of $\mathrm{AI}$ and cloud computing, which fosters the diffusion of AI by providing AI capabilities and guidance in model development, deployment and (re)configuration through the cloud with the objective to increase the affordability and accessibility of AI across industries (Lins et al., 2021). In that, AIaaS also allows organizations with lessadvanced AI development capabilities to access respective resources and integrate them into their own business and IS ecosystems, (Janiesch et al., 2021). However, the increased use of Infrastructure- Platform-, and Software-as-a-Service solutions, on the one hand, facilitates cognitive automation but simultaneously leads to competitive platform ecosystem tensions among ecosystem players. This calls for research in electronic markets on developing novel applications, specifying roles, and innovating business models to thrive and survive against the backdrop of AIaaS and their respective ecosystems (see also Janiesch et al., 2021).

\section{Sourcing- and capability-oriented research opportunities}

Furthermore, organizations are challenged to manage the tradeoff between plug-and-play solutions and highly individualized implementations. Thus, researchers and practitioners alike will face the challenge of developing strategies on how to seamlessly integrate these systems internally while balancing the spectrum of lightweight and heavyweight IT. For instance, research calls for further investigating how to process execution exceptions caused by software robots by routing them to business process management systems for further handling (König et al., 2020). In addition to this technology management perspective, organizations also need to make strategic decisions on whether to build up AI and ML skills and knowledge within the organization vs. outsourcing the latter. As cognitive automation skills are still scarce on the market, this also opens research opportunities for the field of IS education to develop, introduce, and teach new courses to the future or existing workforce, which are tailored to cognitive automation (Herm et al., 2021).

Moreover, organizations need to be facilitated to collect available data on tasks or processes that are subject to cognitive automation in "non-invasive, privacy-preserving manner" internally or seize external vendors of transfer learning services in a commodity-oriented manner, which offers further research opportunities (Herm et al., 2021, p.303). This shows that organizations need to be facilitated to develop and deploy ecosystemic strategies for building up cognitive automation capabilities, which relates to sourcing the required technological and skill-oriented resources for leveraging the steep developments in AIT - i.e., advancements in data storage, algorithms, and computing power - for automation. Furthermore, BPA is predicted to evolve beyond company boundaries facilitating the automation of interorganizational transactions (Lacity \& Willcocks, 2021). In these, the lion's share of project effort has been found to hide in establishing agreements on mutual data standards, governance models, compliance, and intellectual property (Lacity \& Willcocks, 2021). Therefore, this calls for IS research on providing decision-support for respective ecosystemic sourcing strategies, value-cocreation strategies, as well as governance mechanisms. This is particularly suited for research in electronic markets (Alt \& Klein, 2011).

\section{Work system-oriented research opportunities}

Besides IS ecosystem-related challenges, organizations face a variety of socio-technical challenges to leverage cognitive automation for competitive advantage (see also Engel et al., 2021b). Here, organizations are still struggling to gather and process the large amounts of data that are needed to train software robots for cognitive automation and face challenges in the realm of data quality, which is viewed to be one of the major challenges of cognitive automation (Bruckner et al., 2012; Engel et al., 2021b; Lacity \& Willcocks, 2018b; Poosapati et al., 2018). Furthermore, organizations 
are challenged when approaching to transfer algorithmic insights, i.e., ML outcomes, between domains, and face issues in explaining what happens between data input and ML outputs, which leads to the black box character of AI (Lacity \& Willcocks, 2018b). Here, research has emphasized the need for making decisions of cognitive automation tools explainable to business users, which may be approached by developing novel key performance indicators and respective visualizations (Herm et al., 2021). Moreover, there exist challenges in embedding cognitive automation technology in organizations, which refers to the required changes that need to be induced into socio-technical systems (Engel et al., 2021b; Lacity \& Willcocks, 2018b). This opens up various research opportunities:

Like in physical automation, it is important to avoid under- or over-automation when making decisions on cognitive automation initiatives (Fasth-Berglund \& Stahre, 2013). Here, questions arise such as "[h]ow, when, and where should leaders be thinking about applying the various automation technologies to their businesses" (Zarkadakis et al., 2016, p.3). Therefore, substantiated empirical, realworld facts, methods and tool-support are needed to guide the formation of the right cognitive automation strategy. In this realm, case- and design-oriented research is needed on how to select suitable tasks and processes to be automated with cognitive automation, as well as to choose and design the right cognitive automation tools (Poosapati et al., 2018). Through this, practitioners and researchers alike can be supported in successfully planning, developing, embedding, and perpetuating cognitive automation systems in organizations in a manner of technology-driven organizational change ("Technochange") (Markus, 2004). Here, research has emphasized the need to further investigate the single phases of planning, developing, embedding, and perpetuating cognitive automation systems in a more thorough manner, e.g., by developing testing strategies tailored to cognitive automation to increase the success rates of such initiatives (König et al., 2020).

Furthermore, it is necessary to prevent researchers and practitioners from merely layering a new technology on old, unchanged tasks and processes. Against this backdrop, cognitive automation may require an adaptation of respective tasks, processes, and whole business models (Butner $\&$ Ho, 2019). This provides vast potential for IS research to seize its integrative and interdisciplinary nature to help organizations in managing the adaptation of, the interaction between, and the transition from old to new work systems (Bruckner et al., 2012). Thus, cognitive automation will impact how organizations conduct business, and how value creation mechanisms function, which ultimately affects the future of work. As the number of tasks and processes that are candidates for cognitive automation is steadily increasing, the workforce of the future will be required to re-skill workers towards more unique human work (Card \& Nelson, 2019). Consequently, organizations will have to adapt structures and organizational practices and align the new technology with a comprehensive strategy regarding the future of work (Zarkadakis et al., 2016). Thus, future research faces the opportunity to investigate respective proactive workforce training and change management practices (König et al., 2020) to mitigate the risk of employees distrusting cognitive automation systems (Herm et al., 2021). Overall, this shall account for benefitting from the advantages of cognitive automation in a responsible manner. In this vein, we can observe that there are tasks and processes that are neither purely conducted by humans nor purely by cognitive machines. Often, we encounter so-called "hybrid intelligence" (Dellermann et al., 2019) approaches, where the entities - human and machine agent - mutually achieve a higher performance than if they acted separately. Thus, investigating cognitive automation in hybrid work settings, i.e., humans interacting with cognitive automation systems, exhibits vast potential for future research.

\section{Conclusion}

In this fundamental article, we provide an overview of the constituting concepts of cognitive automation. On this basis, we develop an integrated conceptualization of cognitive automation and provide an overview of BPA approaches such as RPA, WfM, and ML-facilitated BPA while pointing out their complementary relationship in the ecosystem of automation solutions. This helps us establish a unified conceptual lens for advancing research on cognitive automation and contribute to a more realistic, less hype- and fear-induced future of work debate regarding cognitive automation. In terms of future research opportunities, the (renewed) rise of AI that facilitates cognitive automation leads to various changes in all socio-technical components of organizations and in IS ecosystems, thus causing large impact on the core of the IS discipline. By extending the deterministic automation logic, which we refer to as "rule-based automation" in this paper, through taking over certain cognitive functions, cognitive automation systems require the adaptation, development, and integration of (new) constructs, models, methods, and theories in IS. This will help to better predict and explain the phenomenon of cognitive automation and the effects of AIT. Ultimately, this shall support managerial decision making on using AI to automate or augment knowledge and service work as well as guide the design of such cognitive automation systems towards valuable, sustainable, and ethical deployment. 


\section{References}

Abdel-Karim, B. M., Pfeuffer, N., \& Hinz, O. (2021). Machine learning in information systems - a bibliographic review and open research issues. Electronic Markets, 31(3). https://doi.org/10. 1007/s12525-021-00459-2.

Alt, R., \& Klein, S. (2011). Twenty years of electronic markets research-looking backwards towards the future. Electronic Markets, 21(1), 41-51. https://doi.org/10.1007/s12525-011-0057-z

Asatiani, A., \& Penttinen, E. (2016). Turning robotic process automation into commercial success - Case OpusCapita. Journal of Information Technology Teaching Cases, 6(2), 67-74. https://doi. org/10.1057/jittc.2016.5

Benlian, A., Kettinger, W. J., Sunyaev, A., \& Winkler, T. J. (2018). Special Section: The Transformative Value of Cloud Computing: A Decoupling, Platformization, and Recombination Theoretical Framework. Journal of Management Information Systems, 35(3), 719-739. https://doi.org/10.1080/07421222.2018.1481634

Bishop, C. M. (2006). Pattern Recognition and Machine Learning. Springer.

Bruckner, D., Zeilinger, H., \& Dietrich, D. (2012). Cognitive Automation-Survey of Novel Artificial General Intelligence Methods for the Automation of Human Technical Environments. IEEE Transactions on Industrial Informatics, 8(2), 206-215. https://doi.org/ 10.1109/TII.2011.2176741

Butner, K., \& Ho, G. (2019). How the human-machine interchange will transform business operations. Strategy and Leadership, 47(2), 25-33. https://doi.org/10.1108/SL-01-2019-0003

Bygstad, B. (2017). Generative innovation: A comparison of lightweight and heavyweight IT. Journal of Information Technology, 32(2), 180-193. https://doi.org/10.1057/jit.2016.15

Card, D., \& Nelson, C. (2019). How automation and digital disruption are shaping the workforce of the future. Strategic HR Review, 18(6), 242-245. https://doi.org/10.1108/shr-08-2019-0067

Coombs, C., Hislop, D., Taneva, S. K., \& Barnard, S. (2020). The strategic impacts of Intelligent Automation for knowledge and service work: An interdisciplinary review. Journal of Strategic Information Systems, 29(4), 1-30. https://doi.org/10.1016/j.jsis. 2020.101600

Davenport, T. H., \& Kirby, J. (2016). Only humans need apply: winners and losers in the age of smart machines. Harvard Business School. Harper Business New York, NY.

Dellermann, D., Ebel, P., Söllner, M., \& Leimeister, J. M. (2019). Hybrid Intelligence. Business and Information Systems Engineering, 61(5), 637-643. https://doi.org/10.1007/s12599-019-00595-2

Drucker, P. F. (1993). Concept of the Corporation. Transaction Publishers.

Engel, C., Ebel, P., \& van Giffen, B. (2021a). Empirically exploring the cause-effect relationships of ai characteristics, project management challenges, and organizational change. 16th International Conference on Wirtschaftsinformatik (pp. 1-17). Essen, Germany.

Engel, C., Elshan, E., \& Ebel, P. (2021b). Deploying a model for assessing cognitive automation use cases: Insights from action research with a leading european manufacturing company. Proceedings of the 54th Hawaii International Conference on System Sciences (pp. 6253-6262).

Fasth-Berglund, A., \& Stahre, J. (2013). Cognitive automation strategy for reconfigurable and sustainable assembly systems. Assembly Automation, 33(3), 294-303. https://doi.org/10.1108/ AA-12-2013-036

Gershenson, C. (2003). On the notion of cognition. ArXiv Preprint Cs/0303006

Ghahramani, Z. (2015). Probabilistic machine learning and artificial intelligence. Nature, 521(7553), 452-459. https://doi.org/10.1038/ nature 14541
Gubrud, M. A. (1997). Nanotechnology and international security. Fifth Foresight Conference on Molecular Nanotechnology (Vol. 1).

Haenlein, M., \& Kaplan, A. (2019). A brief history of artificial intelligence: On the past, present, and future of artificial intelligence. California Management Review, 61(4), 5-14. https://doi.org/10. 1177/0008125619864925

Herm, L. V., Janiesch, C., Reijers, H. A., \& Seubert, F. (2021). From Symbolic RPA to Intelligent RPA: Challenges for Developing and Operating Intelligent Robots. In A. Polyvyanyy, M. T. Wynn, A. Van Looy, \& M. Reichert (Eds.) (pp. 289-305). Cham: Springer International Publishing.

Hofmann, P., Jöhnk, J., Protschky, D., \& Urbach, N. (2020a). Developing purposeful ai use cases - A structured method and its application in project management. Proceedings of the 15th International Conference on Wirtschaftsinformatik. Potsdam, Germany. https:// doi.org/10.30844/wi_2020_a3

Hofmann, P., Samp, C., \& Urbach, N. (2020b). Robotic process automation. Electronic Markets, 30(1), 99-106. https://doi.org/10. 1007/s12525-019-00365-8

Janiesch, C., Fischer, M., Winkelmann, A., \& Nentwich, V. (2019). Specifying autonomy in the Internet of Things: The autonomy model and notation. Information Systems and E-Business Management, 17(1), 159-194. https://doi.org/10.1007/s10257-018-0379-x

Janiesch, C., Zschech, P., \& Heinrich, K. (2021). Machine learning and deep learning. Electronic Markets, 31(3), 685-695. https://doi. org/10.1007/s12525-021-00475-2.

Jordan, M. I., \& Mitchell, T. M. (2015). Machine learning: Trends, perspectives, and prospects. Science, 349(6245), 255-260. https:// doi.org/10.1126/science.aaa8415

Kahneman, D. (2015). Thinking, Fast and Slow. College Music Symposium (Vol. 55). London: Macmillan. https://doi.org/10.18177/ sym.2015.55.ca.10990

König, M., Bein, L., Nikaj, A., \& Weske, M. (2020). Integrating Robotic Process Automation into Business Process Management. In A. Asatiani, J. M. García, N. Helander, A. Jiménez-Ramírez, A. Koschmider, J. Mendling, Mendling, J., Meroni, G. \& H. A. Reijers (Eds.), Lecture Notes in Business Information Processing (Vol. 393 LNBIP, pp. 132-146). Cham: Springer International Publishing. https://doi.org/10.1007/978-3-030-58779-6_9

Kroll, C., Bujak, A., Darius, V., Enders, W., \& Esser, M. (2016). Robotic Process Automation - Robots conquer business processes in back offices. Capgemini Consulting, 1-48. Retrieved from https://www.capgemini.com/consulting-de/wp-content/uploads/ sites/32/2017/08/robotic-process- automation-study.pdf

Lacity, M., \& Willcocks, L. (2018a). Client Service Automation Deployments - What Do They Mean for Your Job and Organization? Pulse Magazine.

Lacity, M., \& Willcocks, L. (2018b). Robotic process and cognitive automation: the next phase. Information and Organization. Ashford, UK: SB Publishing.

Lacity, M., \& Willcocks, L. (2021). Becoming Strategic with Intelligent Automation. MIS Quarterly Executive, 20(2), 1-14. Retrieved from https://standards.ieee.org/

LeCun, Y., Bengio, Y., \& Hinton, G. (2015). Deep learning. Nature, 521(7553), 436-444. https://doi.org/10.1038/nature14539

Lins, S., Pandl, K. D., Teigeler, H., Thiebes, S., Bayer, C., \& Sunyaev, A. (2021). Artificial Intelligence as a Service. Business \& Information Systems Engineering, 63(4), 441-456. https://doi.org/10. 1007/s12599-021-00708-w

Markus, M. L. (2004). Technochange management: Using IT to drive organizational change. Journal of Information Technology, 19(1), 4-20. https://doi.org/10.1057/palgrave.jit.2000002

Neisser, U. (2014). Cognitive psychology: Classic edition. Cognitive Psychology: Classic Edition. Psychology Press. https://doi.org/ $10.4324 / 9781315736174$ 
Nobre, F. S., Tobias, A. M., \& Walker, D. S. (2009). The impact of cognitive machines on complex decisions and organizational change. AI and Society, 24(4), 365-381. https://doi.org/10.1007/ s00146-009-0207-4

Ogiela, L., \& Ogiela, M. R. (2014). Cognitive systems for intelligent business information management in cognitive economy. International Journal of Information Management, 34(6), 751-760. https://doi.org/10.1016/j.ijinfomgt.2014.08.001

Parasuraman, R., \& Riley, V. (1997). Humans and automation: Use, misuse, disuse, abuse. Human Factors, 39(2), 230-253. https:// doi.org/10.1518/001872097778543886

Parasuraman, R., Sheridan, T. B., \& Wickens, C. D. (2000). A model for types and levels of human interaction with automation. IEEE Transactions on Systems, Man, and Cybernetics Part a: Systems and Humans., 30(3), 286-297. https://doi.org/10.1109/3468. 844354

Park, S.-C. (2018). The Fourth Industrial Revolution and implications for innovative cluster policies. AI \& Society, 33(3), 433-445.

Poosapati, V., Manda, V. K., \& Katneni, V. (2018). Cognitive Automation Opportunities, Challenges and Applications. Journal of Computer Engineering and Technology, 9(5), 89-95.

Pramod, A., Naicker, H. S., \& Tyagi, A. K. (2021). Machine Learning and Deep Learning: Open Issues and Future Research Directions for the Next 10 Years. In Computational Analysis and Deep Learning for Medical Care (pp. 463-490). Wiley. https://doi.org/ 10.1002/9781119785750.ch18

Rai, A., Constantinides, P., \& Sarker, S. (2019). Editor's Comments: Next-Generation Digital Platforms: Toward Human-AI Hybrids. Management Information Systems Quarterly, 43(1), iii-ix.

Rainey, S. K., Brown, B., \& Kirk, D. B. (2017). Bots, natural language processing, and machine learning. Tax Executive, 69, 39-46. HeinOnline, https://heinonline.org/HOL/P?h=hein.journ als/taxexe69\&i=357.

Russell, S. J., \& Norvig, P. (2021). Artificial intelligence: A modern approach (Fourth edi). Pearson.

Sampson, S. E. (2021). A Strategic Framework for Task Automation in Professional Services. Journal of Serviec Research, 24(1), 122-140. https://doi.org/10.1177/1094670520940407

Selz, D. (2020). From electronic markets to data driven insights. Electronic Markets, 30(1), 57-59. https://doi.org/10.1007/ s12525-019-00393-4

Stohr, A., \& O'Rourke, J. (2021). Through the cognitive functions lens-a socio-technical analysis of predictive maintenance. 16th International Conference on Wirtschaftsinformatik (pp. 1-16). Essen, Germany.
Syed, R., Suriadi, S., Adams, M., Bandara, W., Leemans, S. J. J., Ouyang, C., Hofstede, A. H. M. ter, van de Weerd, I., Wynn, M. T. \& Reijers, H. A. (2020). Robotic process automation: Contemporary themes and challenges. Computers in Industry, 115, 1-15. https:// doi.org/10.1016/j.compind.2019.103162.

Ter Hofstede, A. H. M., Van Der Aalst, W. M. P., Adams, M., \& Russell, N. (2010). Modern business process automation: YAWL and its support environment. Modern Business Process Automation: YAWL and its Support Environment. Springer Science \& Business Media. https://doi.org/10.1007/978-3-642-03121-2

van der Aalst, W. M. P., Bichler, M., \& Heinzl, A. (2018). Robotic Process Automation. Business \& Information Systems Engineering, 60(4), 269-272. https://doi.org/10.1007/s12599-018-0542-4

Van Der Aalst, W., Van Hee, K. M., \& van Hee, K. (2004). Workflow management: models, methods, and systems. MIT press.

vom Brocke, J., Simons, A., Riemer, K., Niehaves, B., Plattfaut, R., \& Cleven, A. (2015). Standing on the shoulders of giants: Challenges and recommendations of literature search in information systems research. Communications of the Association for Information Systems, 37(9), 205-224. https://doi.org/10.17705/1cais. 03709

von Krogh, G. (2018). Artificial Intelligence in Organizations: New Opportunities for Phenomenon-Based Theorizing. Academy of Management Discoveries, 4(4), 404-409. https://doi.org/10.5465/ amd.2018.0084

Webster, J., \& Watson, R. T. (2002). Analyzing the Past to Prepare for the Future: Writing a Literature Review. MIS Quarterly, 26(2), xiii-xxiii. 10.1.1.104.6570

Wilamowski, B. M., \& Irwin, J. D. (2018). Intelligent systems. CRC Press.

Winfield, A. (2020). Intelligence is not one thing. Journal of Artificial General Intelligence, 11(2), 97-100. https://doi.org/10.2478/ jagi-2020-0003

Zarkadakis, G., Jesuthasan, R., \& Malcolm, T. (2016). The 3 Ways Work Can Be Automated. Harvard Business Review, (October 13), 1-7. Retrieved from https://hbr.org/2016110/the-3-wayswork-can-be-automated

Publisher's note Springer Nature remains neutral with regard to jurisdictional claims in published maps and institutional affiliations. 